\title{
Temporal evolution of fanning in photorefractive materials
}

\author{
Mordechai Segev, Doruk Engin, and Amnon Yariv \\ Department of Applied Physics, California Institute of Technology, Pasadena, California 91125 \\ George C. Valley \\ Hughes Research Laboratories, 3011 Malibu Canyon Road, Malibu, California 90265
}

Received December 7, 1992

\begin{abstract}
We present detailed calculations of the temporal and spatial evolution of beam fanning in photorefractive crystals that is initiated by scattering from noise. We show that fanning starts from beam coupling between the incident radiation and part of the incident radiation scattered by noise at or near the input plane. We show that scattering within the volume of the crystal has negligible effect on fanning, that absorption affects the time response but not the spatial pattern of the fanning, and that the difference between calculations including only phase-matched terms and those including non-phase-matched terms is negligible.
\end{abstract}

Beam fanning is the process of self-induced scattering observed in almost all photorefractive crystals. In its most common manifestation a single beam entering one face of a crystal fans out in the horizontal and/or vertical directions. It is now well established that fanning is an example of stimulated photorefractive scattering and is caused by energyexchange beam coupling between the incident beam and radiation scattered by inhomogeneities in the entrance face or within the bulk of the crystal. ${ }^{1-8}$ The alternative view, that beam fanning is caused by a whole-beam effect in which the trapped charge follows the beam profile while the space-charge field and refractive-index profiles follow a spatial integral of the trapped charge, ${ }^{9-11}$ now seems inapplicable since it fails to predict fanning for an input wave that is a single plane wave and gives fanning that is too small relative to observations for collimated beams with diameters of the order of a millimeter.

Previous calculations of beam fanning led to detailed steady-state results and to limited results of the temporal dependence. ${ }^{5}$ Here we present detailed calculations of the temporal dependence of beam fanning obtained by numerical solutions of the paraxial wave equation for the optical beam and the equation for the time dependence of the refractive-index perturbation produced by interference between the incident beam and radiation scattered by imperfections in the crystal. Our calculations extend the results of Ref. 5 for steady-state fanning to temporal evolution and include the effects of absorption and Rayleigh scattering distributed throughout the crystal.

We formulate the problem of beam propagation in photorefractive materials by using the planewave expansion technique that we have recently used for stimulated photorefractive backscattering ${ }^{12}$ and for counterpropagating beams. ${ }^{13}$ Similar expansions have been used previously for fanning by Kukhtarev $^{14}$ and by Obukhovskii and Stoyanov. ${ }^{2}$ Limitations inherent in this technique are discussed by Crosignani et al. ${ }^{15}$ To isolate the physics of beam fanning alone, we neglect interactions between orthogonal components (anisotropic scattering), as- sume a monochromatic incident beam and stationary scattering centers, and include only two spatial dimensions.

The electric field $E(x, z, t)$ of the optical beam propagating in the $z$ direction may be written in the form

$$
\begin{aligned}
& E(x, z, t)=\frac{1}{2}[\exp (i k z-i \omega t) A(x, z, t)+\text { c.c. }], \\
& A(x, z, t)=\sum_{m} a_{m}(z, t) \exp [-i k \epsilon m(x+\epsilon m z / 2)],
\end{aligned}
$$

where $A(x, z, t)$ is the slowly varying part of the field, $x$ is the transverse coordinate, $k=\omega n_{b} / c$ is the optical wave number, $n_{b}$ is the background refractive index in the crystal, $\epsilon$ is the angular separation of the plane waves, and $a_{m}(z, t)$ are the expansion coefficients. The amplitude $A$ obeys the paraxial wave equation

$$
\frac{\partial A}{\partial z}-\frac{i}{2 k} \frac{\partial^{2} A}{\partial x^{2}}+\frac{\alpha A}{2}=\frac{i k}{n_{b}} \delta n(x, z, t) A,
$$

where the refractive-index perturbation $\delta n(x, z, t)$ is determined by the interference of the individual plane waves and $\alpha$ is the absorption coefficient. The equation for $\delta n$ may be written as

$$
\begin{aligned}
\frac{\partial \delta n}{\partial t}+ & \left(1+|A|^{2}\right) \delta n=i \sum_{m, n} \gamma_{m n} a_{m} a_{n}{ }^{*} \\
& \times \exp \left[-i k(m-n) x \epsilon-i k \epsilon^{2}\left(m^{2}-n^{2}\right) z / 2\right],
\end{aligned}
$$

where $\gamma_{m n}$ are the coupling coefficients between the individual plane waves as given in Ref. 5 . Here we assume that $\gamma_{m n}$ are real (no applied fields or internal photovoltaic fields) since beam fanning is an energytransfer process caused by the nonlocal (or real) part of the coupling coefficients. In Eqs. (1)-(4) the field amplitudes, $A$ and $a_{n}$, are given in units of the square root of the equivalent dark irradiance (the optical irradiance necessary to produce a carrier density equal to the dark carrier density) and time is in units of the dielectric relaxation time evaluated at the equivalent dark irradiance (see Ref. 12). The 
refractive-index perturbation $\delta n(x, z, t)$ may also be expanded in the form

$$
\begin{aligned}
& \delta n(x, z, t)=\sum_{m, n} n_{m, n}(z, t) \\
& \times \exp \left[i k(m-n) x \epsilon-i k \epsilon^{2}\left(m^{2}-n^{2}\right) z / 2\right]
\end{aligned}
$$

In what follows, we neglect the transverse dependence of the $|A|^{2}$ term on the left-hand side of Eq. (4). This gives a good approximation when the mean intensity is nearly independent of the transverse direction (e.g., a single incident plane wave or an unfocused beam of large cross section combined with conditions in which the fanned intensity is small compared with the incident intensity). It is also a good approximation if the modulation index of the interference pattern of all the plane waves is small compared to unity, since the spatial dependence is second order in the modulation index. One situation in which the spatial dependence of the $|A|^{2}$ term cannot be neglected is that of a tightly focused beam, which was considered in Ref. 5 for steady-state conditions.

Substitution of Eqs. (1), (2), and (5) into Eqs. (3) and (4), multiplication by the factor $\exp (i k p x \epsilon)$, and integration over $x$ yields

$$
\begin{gathered}
\frac{\partial a_{p}}{\partial z}+\frac{\alpha a_{p}}{2}=\frac{i k}{n_{b}} \sum_{m, n} n_{m n} a_{p+n-m} \exp \left(-i k \epsilon^{2} \Delta z / 2\right), \\
\frac{\partial n_{m n}}{\partial t}+I_{0} n_{m n}=\gamma_{m n} a_{m} a_{n}^{*},
\end{gathered}
$$

where $I_{0}$ is the spatial average of $1+|A|^{2}, \Delta=2(m-$ $n)(m-p)$, and $k \epsilon^{2} \Delta / 2$ is the momentum mismatch between a specific plane wave $a_{p}$ and the grating $n_{m n}$. Including only the phase-matched terms in the summation in Eq. (6) yields the simpler equation (recall that $\gamma_{m m}=0$ ):

$$
\frac{\partial a_{p}}{\partial z}+\frac{\alpha a_{p}}{2}=\frac{i k}{n_{b}} \sum_{n} n_{p n} a_{n}
$$

For steady-state conditions, substitution of Eq. (7) into Eq. (8) yields Eq. (1) of Ref. 5. In the calculations that follow, we use the same parameters as in Ref. 5, which are typical for $\mathrm{BaTiO}_{3}$ crystals and provide a good match to the steady-state observations. Angles are measured with respect to the $+c$ axis, and 21 plane waves are used to describe the transverse dependence (angular separation of $1^{\circ}$ ). In our calculations, we have used the numerical methods of Ref. 12, which are explained in detail therein. We have carried out calculations with as many as 81 plane waves and shown that the differences between 21 and 81 plane-wave calculations are small. Since our calculations show that non-phasematched terms are important only for propagation distances of a few optical wavelengths, all our results are based on a numerical solution of Eqs. (7) and (8).

We first obtain the time dependence of fanning for a crystal in which absorption is negligible and in which the fanning is seeded by inhomogeneities in the input facet (i.e., no volume scattering). The initial conditions assume a single plane wave of intensity $I_{0}=1$ (in units of the equivalent dark irradiance) incident at $14^{\circ}$ with respect to the $+c$ axis and 20 noise plane waves with an intensity of $10^{-4} I_{0}$ (i.e., $a_{n}=10^{-2}$, and a uniform transverse phase). Figure 1 shows the evolution of the fanning amplitude in the Fouriertransform plane of the crystal output (which can be regarded as the pattern in the far field of the crystal) for a crystal thickness of $L=0.5 \mathrm{~cm}$. Note that it takes approximately 10 units of time to reach steady state and that the pattern obtained in the steady state is identical to that obtained in Ref. 5 .

We added nonzero absorption to the calculation of fanning and obtained a pattern that is virtually identical to that shown in Fig. 1, but the time to reach steady state is approximately $40 \%$ longer for an absorption coefficient $\alpha=1 \mathrm{~cm}^{-1}$. This result can also be shown analytically by setting $a_{p}{ }^{\prime}=a_{p} \exp (\alpha z / 2)$ and $t^{\prime}=t \exp (-\alpha z)$.

We have also compared two alternative models for the noise that starts the fanning process: surface scattering at the input face and volume scattering distributed throughout the crystal. The volume scattering is modeled by adding a term $\sigma_{p}$ (in inverse centimeters) to the right-hand side of Eq. (8). Integration of Eq. (8) along the direction of propagation shows that this term contributes an amount $\sigma_{p} L$ to $a_{p}$. We have performed calculations for $\sigma_{p}=$ $7 \times 10^{-3} \mathrm{~cm}^{-1}$ and $L=0.5 \mathrm{~cm}$ for comparison with the $a_{p}(0)=10^{-2}$. As Fig. 2 shows, there is little difference between starting the fanning from surface scattering or volume scattering at these levels. Direct comparison of the two levels is a little problematical since the surface scattering has a single phase front while the volume scattering has a phase that varies throughout the medium and that is filtered by the phase-matching condition. We estimate that volume scattering of a level $\sigma_{p} L=0.35 \times 10^{-3}$ as placed here in the phase-matched equations would be approximately $2 \pi / 0.1$ larger if it were placed in the equations that contain the non-phase-matched terms (assuming that phase matching occurs within an interval of $\sim 0.1 \mathrm{rad}$ ). While adding volume scattering of this level has little effect on the fanning, doubling the surface scattering level $\left[a_{p}(0)=2 \times\right.$ $10^{-2}$ ] completely changes the angular distribution as

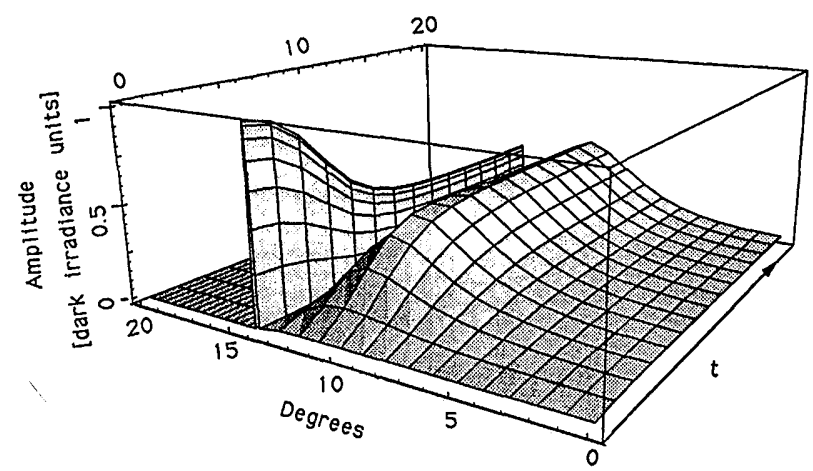

Fig. 1. Temporal evolution of fanning at the output of a photorefractive crystal characterized by parameters that are typical of $\mathrm{BaTiO}_{3}$, with no absorption and an inhomogeneous input surface. We plot amplitude versus angle (degrees) with normalized time (as defined in the text). The spike corresponds to the far field of the incident plane wave. 


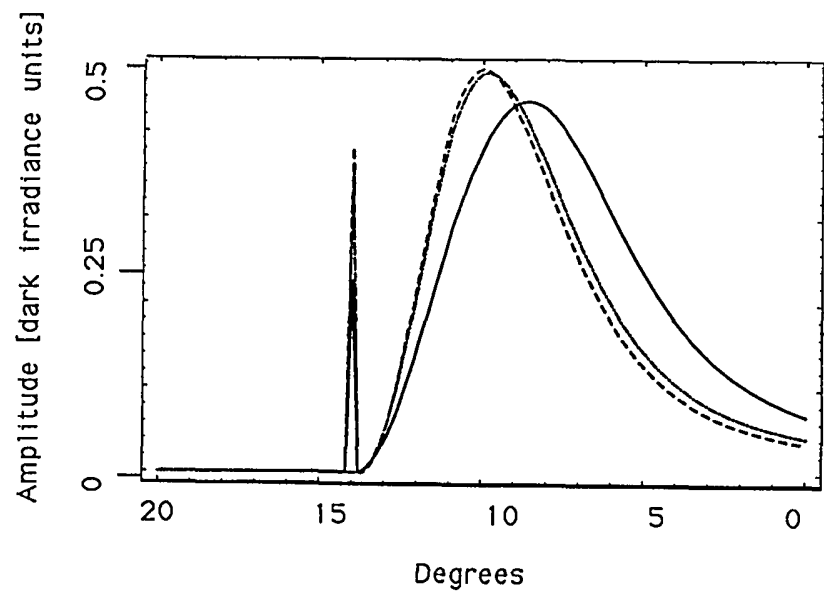

Fig. 2. Steady-state fanning at the output of a photorefractive crystal characterized by parameters that are typical of $\mathrm{BaTiO}_{3}$ with zero absorption and seeded by varying noise conditions. The dashed curve is the baseline case with the crystal seeded by surface scattering, the curve next to it is with the crystal seeded by an inhomogeneous input surface and by scattering distributed throughout the volume, and the solid curve is with the crystal seeded by twice as much surface scattering.

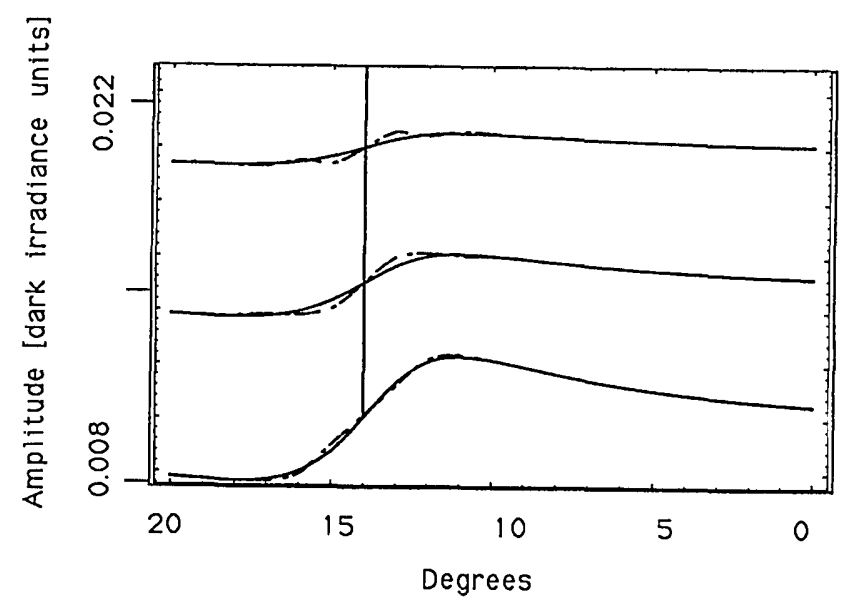

Fig. 3. Comparison of the plane-wave amplitudes after propagation of $50 \mu \mathrm{m}$ (upper curves), $100 \mu \mathrm{m}$ (middle curves), and $200 \mu \mathrm{m}$ (lower curves) with (dashed curves) and without (solid curves) non-phase-matched terms in the wave equation.

shown by the solid curve in Fig. 2. This feature of the surface scattering has been used to initiate fanning in a $\mathrm{KNbO}_{3}$ crystal. ${ }^{16}$

Note that the inclusion of the volume-scattering term by adding $\sigma_{p}$ to Eq. (8) seems to add energy to the wave-mixing process. This is, of course, unphysical since the scattered light originates from the incident beam, and the additional energy comes from an effective loss to all the plane-wave components. Since all the spatial components are scattered in the same manner, one may compensate for this with an additional loss in the absorption coefficient $\alpha$, which slightly increases from its original value. We have shown above that the loss does not affect the steadystate spatial distribution of the fanning and only lengthens its time response. The representation of the volume scattering by $\sigma_{p}$ in the steady state is therefore accurate and is a very good approximation for the temporal evolution.

Finally, we compared numerical solutions to the steady-state equations with and without non-phasematched terms for $L=50,100,200 \mu \mathrm{m}$. The results in Fig. 3 show the solutions with non-phase-matched terms (dashed curves) and with phase-matched terms (solid curves) for no absorption and noise only at the input facet. Note that by $200 \mu \mathrm{m}$ the two solutions nearly overlay, with the exception of a small ripple. For typical propagation paths of a few millimeters in photorefractive crystals, we conclude that the non-phase-matched terms have little effect on the behavior of beam fanning. An important issue is now the influence of the initial phase, which can be random, on the fanning. It can be shown straightforwardly that, in the temporal steady state, the phase-matched (and therefore the total, including the non-phase-matched part) behavior is dependent on $\left|a_{n}\right|^{2}$, and hence the initial phase of each input (noise) plane-wave component is maintained throughout the process. Therefore the fanning amplitude components at the crystal output exhibit phases identical to the phase of input noise at each individual direction.

The research of Mordechai Segev, Doruk Engin, and Amnon Yariv was supported by the Defense Advanced Research Projects Agency.

\section{References}

1. V. V. Voronov, I. R. Dorosh, Y. S. Kuz'minov, and N. V. Tkachenko, Kvantovaya Elektron. (Moscow) 7, 2313 (1980) [Sov. J. Quantum Electron. 10, 1346 (1980)].

2. V. V. Obukhovskii and A. V. Stoyanov, Kvantovaya Elektron. (Moscow) 12, 563 (1985) [Sov. J. Quantum Electron. 15, 367 (1985)].

3. R. A. Rupp and F. W. Drees, Appl. Phys. B 39, 223 (1986).

4. G. Zhang, Q. X. Li, P. P. Ho, S. Liu, Z. K. Wu, and R. R. Alfano, Appl. Opt. 25, 2955 (1986).

5. M. Segev, Y. Ophir, and B. Fischer, Opt. Commun. 77, 265 (1990).

6. G. C. Valley, J. Opt. Soc. Am. B 4, 14 (1987); erratum, 4, 934 (1987).

7. T. R. Moore and D. L. Walters, J. Opt. Soc. Am. B 5, 1806 (1988).

8. G. C. Valley and W. P. Brown, "Kinky beam paths inside photorefractive crystals," J. Opt. Soc. Am. B (to be published).

9. S. R. King, T. S. Hartwick, and A. B. Chase, Appl. Phys. Lett. 21, 312 (1972).

10. J. Feinberg, J. Opt. Soc. Am. 72, 46 (1982).

11. P. P. Banerjee and J. J. Liu, in Nonlinear Optics: Materials, Fundamentals, and Applications, Vol. 18 of 1992 OSA Technical Digest Series (Optical Society of America, Washington, D.C., 1992), p. 100.

12. G. C. Valley, J. Opt. Soc. Am. B 9, 1442 (1992).

13. M. Segev and A. Yariv, Opt. Lett. 16, 1938 (1991).

14. N. V. Kukhtarev, Kvantovaya Elektron. (Moscow) 8, 1452 (1981) [Sov. J. Quantum Electron. 11, 878 (1981)].

15. B. Crosignani, M. Segev, D. Engin, P. DiPorto, A. Yariv, and G. Salamo, J. Opt. Soc. Am. B 10, 446 (1993).

16. P. Günter, E. Voit, M. Z. Zha, and J. Albers, Opt. Commun. 55, 210 (1985). 